\title{
A Path to the Stars: The Evolution of the Species
}

\author{
Fabrizio Vitali, ${ }^{1}$ Guido Chincarini, ${ }^{2,3}$ Mario Zannoni, ${ }^{2}$ Stefano Covino, ${ }^{3}$ Emilio Molinari, ${ }^{3,4}$ \\ Stefano Benetti, ${ }^{5}$ Carlotta Bonoli, ${ }^{5}$ Favio Bortoletto, ${ }^{5}$ Enrico Cascone, ${ }^{6}$ Rosario Cosentino, ${ }^{4}$ \\ Francesco D'Alessio, ${ }^{1}$ Paolo D'Avanzo, ${ }^{2,3}$ Vincenzo De Caprio, ${ }^{3}$ Massimo Della Valle,,${ }^{6,7}$ \\ Alberto Fernandez-Soto, ${ }^{8}$ Dino Fugazza, ${ }^{3}$ Enrico Giro, ${ }^{5}$ Demetrio Magrin, ${ }^{5}$ \\ Giuseppe Malaspina, ${ }^{3}$ Lech Mankiewicz, ${ }^{9}$ Raffaella Margutti, ${ }^{2}$ Ruben Mazzoleni, ${ }^{3}$ \\ Luciano Nicastro, ${ }^{10}$ Alberto Riva, ${ }^{11}$ Marco Riva, ${ }^{12}$ Ruben Salvaterra, ${ }^{2}$ Paolo Spanò, ${ }^{3}$ \\ Monica Sperandio, ${ }^{3}$ Mauro Stefanon, ${ }^{8}$ Gino Tosti, ${ }^{13}$ and Vincenzo Testa ${ }^{1}$
}

${ }^{1}$ Istituto Nazionale di Astrofisica, Osservatorio Astronomico di Roma, Via Frascati, 33, Monte Porzio Catone, 00040 Roma, Italy ${ }^{2}$ Dipartimento di Fisica G. Occhialini, Università degli Studi di Milano - Bicocca, Piazza della Scienza 3, 20126 Milano, Italy

${ }^{3}$ Istituto Nazionale di Astrofisica, Osservatorio Astronomico di Brera, via Bianchi 46, 23807 Merate, Italy

${ }^{4}$ Istituto Nazionale di Astrofisica, Telescopio Nazionale Galileo, Rambla José Ana Fernández Pérez, 7, 38712 Breña Baja, Spain

${ }^{5}$ Istituto Nazionale di Astrofisica, Osservatorio Astronomico di Padova, Vicolo dell'Osservatorio, 5, 35122 Padova, Italy

${ }^{6}$ Istituto Nazionale di Astrofisica, Osservatorio Astronomico di Capodimonte, Salita Moiariello, 16, 80131 Napoli, Italy

${ }^{7}$ European Southern Observatory, Karl-Schwarzschild-Strasse 2, 85748 Garching bei München, Germany

${ }^{8}$ Instituto de Fisica de Cantabria (CSIC-UC), Edificio Juan Jorda, Av. de los Castros s/n, 39005 Santander, Spain

${ }^{9}$ Center for Theoretical Physics of Polish Academy of Science, Al. Lotnikow 32/46, 02-668 Warsaw, Poland

${ }^{10}$ Istituto Nazionale di Astrofisica, IASF Bologna, via Gobetti 101, 40129 Bologna, Italy

${ }^{11}$ Istituto Nazionale di Astrofisica, Osservatorio Astronomico di Torino, Via Osservatorio, 20, 10025 Pino Torinese, Italy

${ }^{12}$ Dipartimento Ingegneria Aerospaziale, Politecnico Milano, Via La Masa, 34, 20156 Milano, Italy

${ }^{13}$ Dipartimento di Fisica, Facoltà di Scienze MM. FF. NN., Università degli Studi di Perugia, via A. Pascoli, 06123 Perugia, Italy

Correspondence should be addressed to Fabrizio Vitali, vitali@oa-roma.inaf.it

Received 30 June 2009; Accepted 12 January 2010

Academic Editor: Joshua S. Bloom

Copyright (C) 2010 Fabrizio Vitali et al. This is an open access article distributed under the Creative Commons Attribution License, which permits unrestricted use, distribution, and reproduction in any medium, provided the original work is properly cited.

During the last years, a number of telescopes have been dedicated to the followup of the GRBs. But after the Swift launch, the average observed intensity of the GRBs showed to be lower than thought before. Our experience with the robotic $60 \mathrm{~cm}$ REM telescope confirmed this evidence, with a large number of lost GRBs. Then, we proposed to study the feasibility of a $4 \mathrm{~m}$ fast pointing class telescope, equipped with a multichannel imagers, from Visible to Near Infrared. In this paper, we present the main result of the feasibility study we performed so far.

\section{Introduction}

Since the discovery of a dynamic Universe on time scales of hours and seconds it became of paramount importance to have a high sampling frequency of the data. The new epoch started in the late fifties, early sixties with the discovery of short periods in cataclysmic variables. Later on were discovered the X-ray and Gamma-ray radiation very likely due to emissions related to the presence of degenerate matter. Accretion on neutron stars and black holes generate also signals of very short duration, milliseconds and some time even less, randomly distributed in space and time. These bursts of light made it mandatory to have fast response detectors and fast moving telescopes to detect the event during birth and subsequent rapid evolution: astronomers need large apertures and very efficient instruments to detect the faintest signals and large and efficient telescopes reacting immediately to any alert. In 2003, in the frame of the SWIFT project (that does not provide any coverage in the infrared domain), our group designed and realized the $60 \mathrm{~cm}$ robotic visible-infrared 
telescope REM, installed in the La Silla mountain, hosted by the ESO facility. Even if the telescope showed to be a very nice robotic machine and gave excellent scientific results, it resulted too small for the weak burst of ligth coming from the depth of the Universe. Then, we decided to exploit the very positive experience made with REM. We present here the feasibility study of a robotic $4 \mathrm{~m}$ class telescope able to point and track everywhere in the visible sky in 30-50 s, equipped with multichannel VIS and NIR imagers, allowing simultaneous observations from 0.4 to $2.5 \mu \mathrm{m}$, and visible spectrometer and polarimeter ancillary instruments.

\section{The REM Experience}

REM is a $60 \mathrm{~cm}$ robotic telescope, that has been operating on the La Silla mountain (Chile) since 2003, equipped with a visible imager and spectrometer (ROSS) and a NIR imaging camera (REMIR) [1]. During these years, REM acted as a fruitful laboratory, in which we could operate and learn how to control and manage a remote and automatic telescope: the average response time of the REM telescope to a satellite trigger is $\sim 30 \mathrm{~s}$, simultaneous Visible and NIR images can be obtained and all the frames (scientific and technical), all the telescope and instruments parameters are delivered to a database server, which can be queried remotely.

Despite of its small dimensions, REM delivered (and it is still delivering) high quality scientific results, as witnessed, that is, by the two GRBs 060418 and 060607A (see Figure 1), from which a value of the Lorenz factor $\Gamma \approx 400\left(\eta_{0.2} n_{0}\right)^{-1 / 8}$ has been derived [2].

\section{Not Simply a Larger REM}

The precious REM experience, allowed us to think about a larger telescope, able to replicate the same performances in terms of fastness and reliability. Then, we asked and received funds from the Italian Research Ministry and Istituto Nazionale di Astrofisica for CODEVISIR (Conceptual Design for a VISible and nIR telescope), a feasibility study to build and operate a fully robotic $4 \mathrm{~m}$ class telescope, equipped with VIS and NIR instruments, able to react to a satellite trigger in less than $50 \mathrm{~s}$ (with a goal of $30 \mathrm{~s}$ ). We also applied to the ERC FP7 Call for Ideas on Research, Technological Development and Demonstration-Advanced Grant, for PATHOS (PATH to the Stars).

Taking into account of new scientific hints, we decided to change the instrumental configuration with respect to REM, passing to a multichannel approach. Then, the new instruments will be able to collect simultaneous images in the visible $g, r, i, z$ and NIR $J, H$ and $K$ filters. As for REM, the primary science driver is the follow-up of the GRBs, with the main goal to record the early $\left(t-t_{0}<50 \mathrm{~s}\right)$ phase light curve with high temporal resolution and simultaneous multiband imaging, to allow the derivation of the GRB spectral energy distributions and Photo- $z$, even for high redshift GRBs: as the naked eye GRB 080319B has shown [3], micro-variability (sub sec time-scale) during afterglow prompt, that is, in the first tens of seconds, can be a powerful diagnostic tools of
TABLE 1: Configurations.

\begin{tabular}{ll}
\hline Alt-Az & Alt-Alt \\
\hline (i) Blind zenith & (i) Low horizon blind angle \\
(ii) Unbalanced azimuth & (ii) Equilibration on both \\
motion & alt-motions \\
(iii) Off the shelf & (iii) To be developed \\
\hline
\end{tabular}

the physical conditions in the emitting region. Moreover, spectroscopy and polarimetry should be performed for the more brilliant GRBs.

When not observing GRBs, the telescope will be devoted to ancillary science programs, spanning from Supernovae to X-Ray Binaries, from Active Galactic Nuclei to Stellar Evolution and Star Formation.

\section{The Challenge of the Telescope}

The first issue to solve is to build a fast-moving, fully robotic, $4 \mathrm{~m}$ class telescope, for which the main requirements are

(i) pointing rapidity TOO: Required $60^{\circ} / 50 \mathrm{~s}$, Goal $60^{\circ} / 30 \mathrm{~s}$

(ii) pointing Accuracy: 2-3 arcsec;

(iii) tracking error: $0.2 \mathrm{arcsec} / 10 \mathrm{~min}$;

(iv) minimum Corrected Field of view (FOV): $15^{\prime}$;

(v) max PSF degradation on axis: $0.3 \operatorname{arcsec}$ (Active optics, damping and vibration suppression);

(vi) movements Ranges: Azimuth: $\pm 270^{\circ}$, Alt: $\pm 90^{\circ}$, Derotator: $360^{\circ}$.

4.1. Telescope Configuration. We firstly analyzed the best telescope mounting configuration between a classic Alt-z and a less common Alt-Alt (Figure 2). Both configurations have their pros and cons (see Table 1.)

The analysis has shown that the blind zone is not a concern, then, at the moment, we plan to adopt the classical Alt-Az mounting, that has also considerable less costs in term of developing.

4.2. Instrument Location (Nasmyth). We studied also the influence of the instrument location on the overall telescope movements (Figure 3). In Table 2, a comparative study is reported. Currently, our interest is in the Cassegrain configuration, because of the reduced momenta of inertia and minor unbalanced factor.

4.3. The Optical Design. The basic telescope design is based on a classical Ritchey-Chretien, with a F/10 equivalent focal ratio (Figure 4). Primary focal ratio is relatively fast, being $F / 1.5$, to keep the telescope structure very compact, minimizing moments and overall mass. The unusual location of the focal plane, a bent Cassegrain below the primary mirror, has been selected to allow placement of the dichroic train before the focal plane itself. The flat M3 mirror can rotate, to feed different focal stations. 

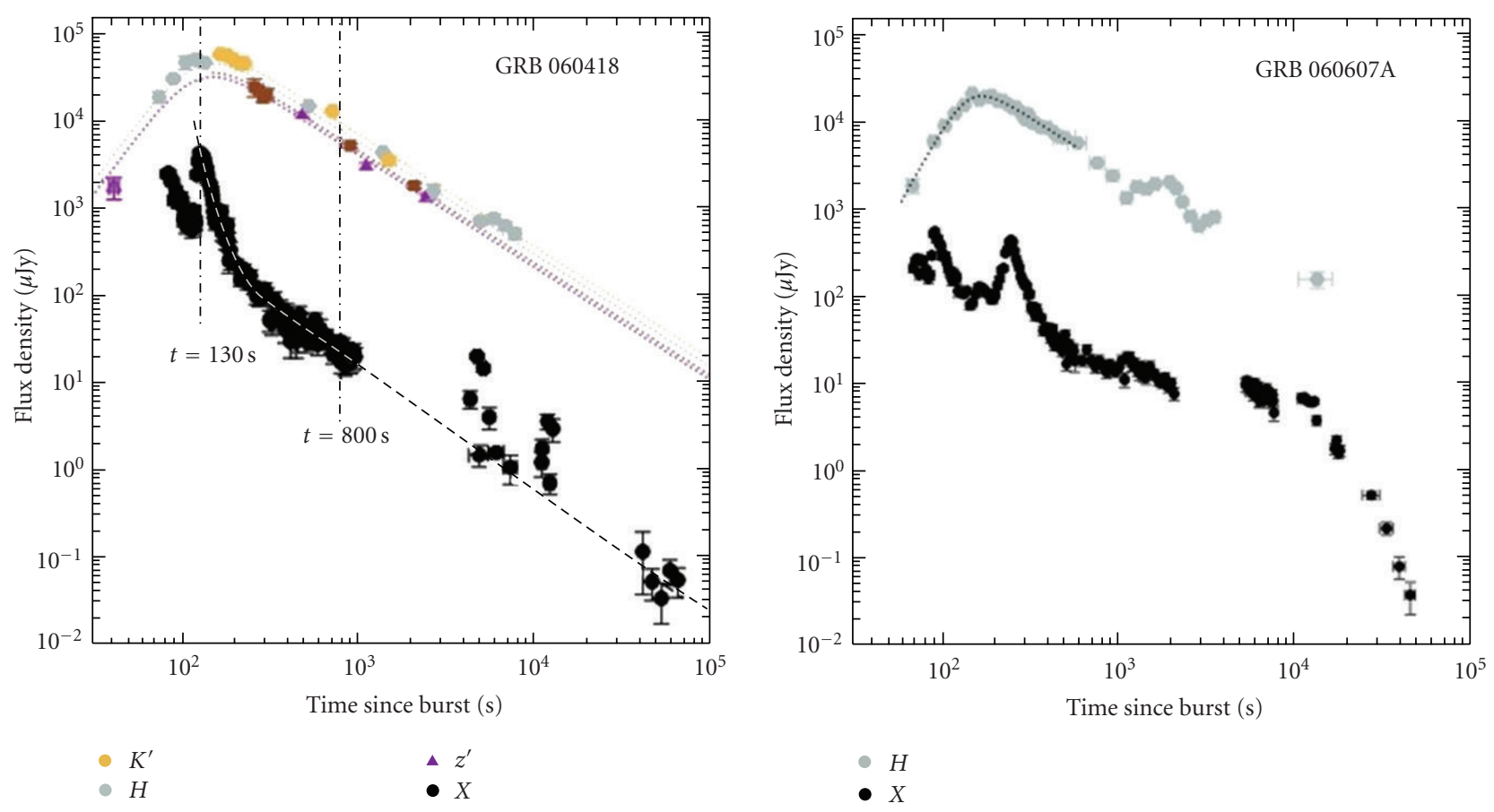

(a)

(b)

FIGURE 1: NIR and X-ray light curves for GRB 060418 and GRB 060607A.

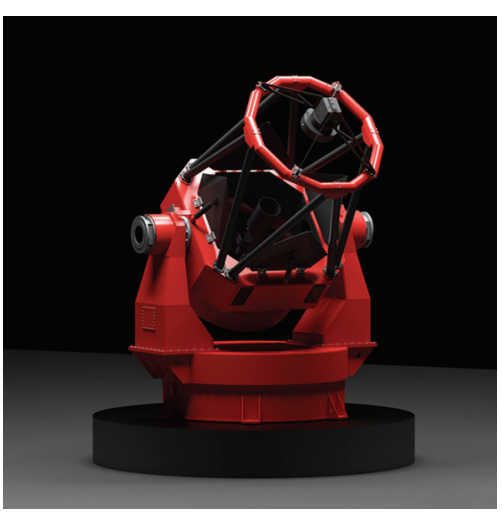

(a)

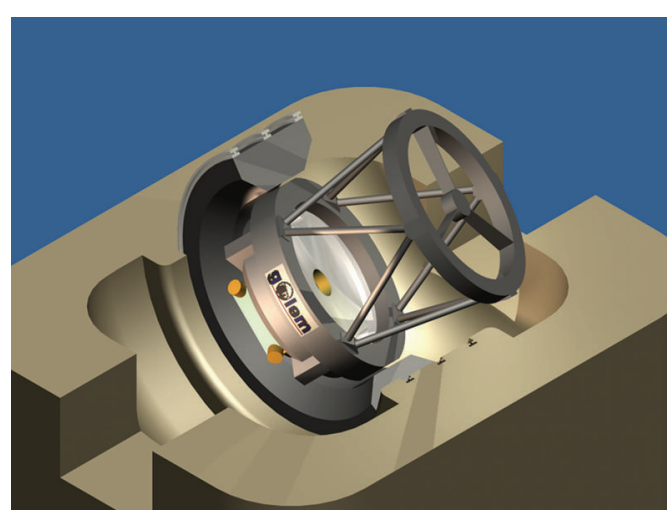

(b)

Figure 2: The Alt-Az (courtesy by Peter Aniol, Astelco) and Alt-Alt telescope configurations.

TABLE 2: A comparison between the two available foci, Nasmyth and Cassegrain.

\begin{tabular}{|c|c|c|c|}
\hline \multicolumn{2}{|c|}{ Nasmyth } & \multicolumn{2}{|c|}{ Cassegrain } \\
\hline \multicolumn{2}{|c|}{ M3 between $\mathrm{M} 1$ and M2 } & \multicolumn{2}{|c|}{ No M3 and mountings between M1 and M2 } \\
\hline \multicolumn{2}{|c|}{ Higher moments of inertia } & \multicolumn{2}{|c|}{ M1 \& Instrs’ reduced moments of inertia } \\
\hline \multicolumn{2}{|c|}{ Only two (probably one) derotation } & \multicolumn{2}{|c|}{ Required a further derotation } \\
\hline \multicolumn{2}{|c|}{ Mass (El stru. + Instr.): $69823 \mathrm{Kg}$} & \multicolumn{2}{|c|}{ Mass (El stru. + Instr.) M: $60127 \mathrm{Kg}$} \\
\hline \multirow{2}{*}{ Elevation $0^{\circ}$} & Izz: $174000 \mathrm{Kgm}^{2}(\mathrm{Az})$ & \multirow{2}{*}{ Elevation $0^{\circ}$} & Izz: $128000 \mathrm{Kgm}^{2}(\mathrm{Az})$ \\
\hline & Ixx: $229000 \mathrm{Kgm}^{2}$ (Alt) & & Ixx: $161000 \mathrm{Kgm}^{2}$ (Alt) \\
\hline \multirow{2}{*}{ Elevation $90^{\circ}$} & Izz: $258000 \mathrm{Kgm}^{2}(\mathrm{Az})$ & \multirow{2}{*}{ Elevation $90^{\circ}$} & Izz: $160000 \mathrm{Kgm}^{2}(\mathrm{Az})$ \\
\hline & Ixx: $229000 \mathrm{Kgm}^{2}$ (Alt) & & Ixx: $161000 \mathrm{Kgm}^{2}$ (Alt) \\
\hline \multicolumn{2}{|c|}{ Unbalance Factor $\left(\mathrm{Az} 0^{\circ} / 90^{\circ}\right): 1,48$} & \multicolumn{2}{|c|}{ Unbalance Factor Izz $\left(0^{\circ} / 90^{\circ}\right): 1,25$} \\
\hline
\end{tabular}




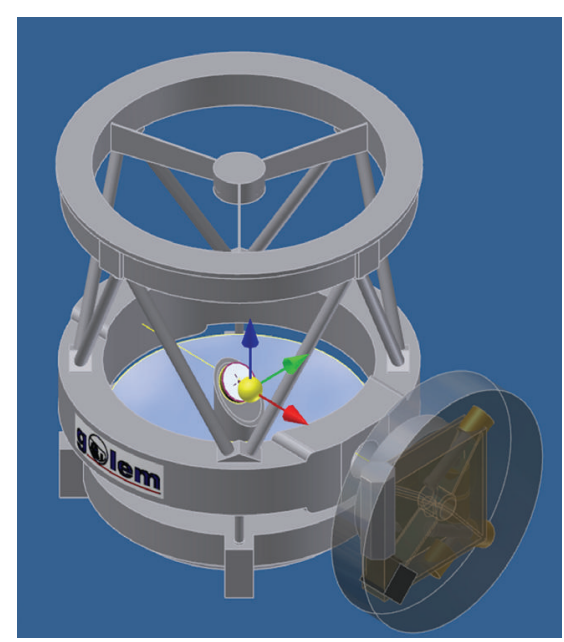

(a)

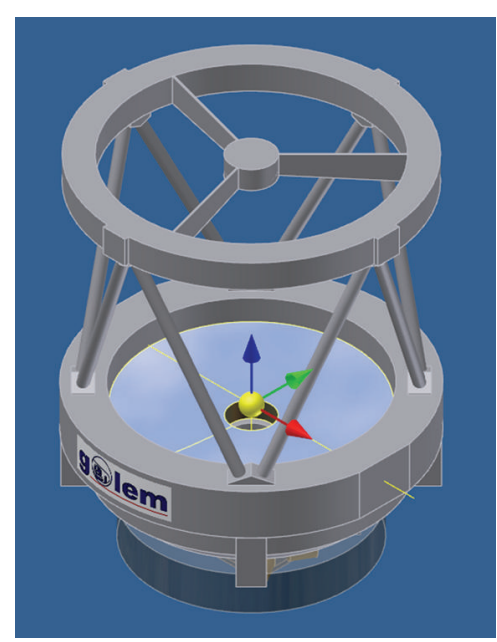

(b)

Figure 3: The two Nasmyth (a) and Cassegrain (b) Instrument Location.

\section{The Instrumentation Suite}

Pathos will be equipped with an instrumentation suite able to cover the wavelength range from the Visible (VIS, $400 \mathrm{~nm}-$ $900 \mathrm{~nm}$ ) to the Near Infrared (NIR, 1.00-2.5 $\mu \mathrm{m}$ ) during the same exposure. This will be allowed by a multichannel imaging configuration, that envisages a detector for each photometric band, delivered through a dichroic cascade along the optical path. The availability of large format arrays, both in the visible and in the infrared wavelength range allows a large flexibility in the optical design, that will ensure a Corrected Field of View of about $10 \mathrm{arcmin}$ for both cameras.

Two ancillary instruments will complete the instrumentation suite, a visible spectrograph and a photopolarimeter, fed by rotating the M3 mirror.

The instrument set up has been thought by integrating optical and mechanical requirements. The mechanical design will be driven mainly by four main requirements:

(i) extreme light weighting of the structure (the fast pointing nature of the telescope),

(ii) weight distribution (same reason as above),

(iii) automation requirement,

(iv) reliability.

Some complementary items must also be taken into account:

(i) thermal stabilization,

(ii) maintenance.

The light weighting is the predominant aspect that will be investigated, both for reducing the overall mass (centrifugal and earthquake passive induced accelerations) and for reducing the thermal inertia of the overall system.
The weight distribution is very critical because of the telescope fast motion requirement. An unbalanced system will induce undesired passive flexions and torsions to the rotating bearings and must be avoided. The best solution is to balance the system just with the payload, (i.e., design of the optical system, location of the nonstructural masses like electronic racks and cables).

The telescope will be remote-managed, that means that all the possible failures must be avoided via proper solutions/redundancy. This implies also an optimization of the maintainability items like handling, accessibility, minimizing of servo-systems, because the absence of the dedicated personnel impose high repairing costs.

The instruments carousel will be organized into 3 systems:

(1) Multi Color Camera (MCC), VIS camera $(g, r, i$, and $z$ bands) plus NIR camera $(J, H$ and $K)$,

(2) Spectrograph (SPEC),

(3) Fast PhotoPolarimeter (FPP).

The MCC will be continuously fed by the telescope beam (see Figure 5), whereas SPEC and FPP will be selected by properly moving the M3 mirror.

The main idea that is driving the MCC is the simultaneous acquisition. This can be done by considering a single detector for each band $(g, r, i, z, J, H$ and $K$, see below for a more detailed description). The infrared part of the instrument will be hosted in a cryogenic environment, to avoid the thermal background and to minimize the level of the detectors dark current.

The optical designs of the visible and infrared arms of the MCC are identical, thus allowing a modular design for the opto-mechanical supports and significantly reducing the design-manufacturing costs. 


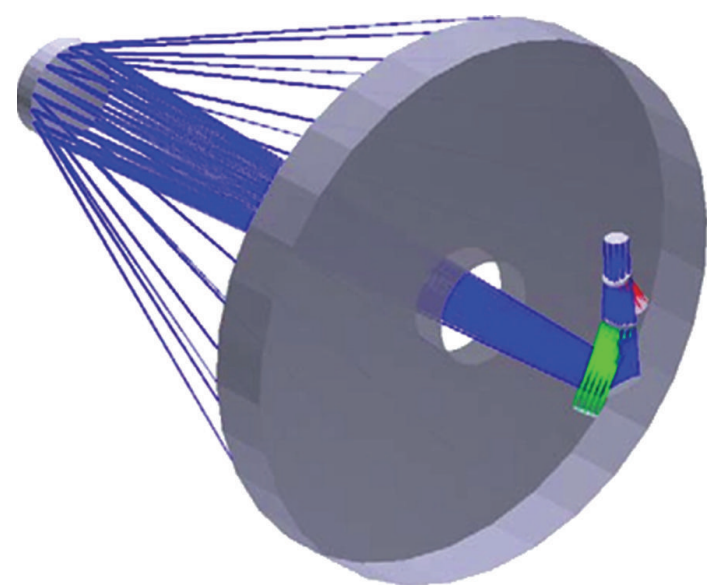

(a)
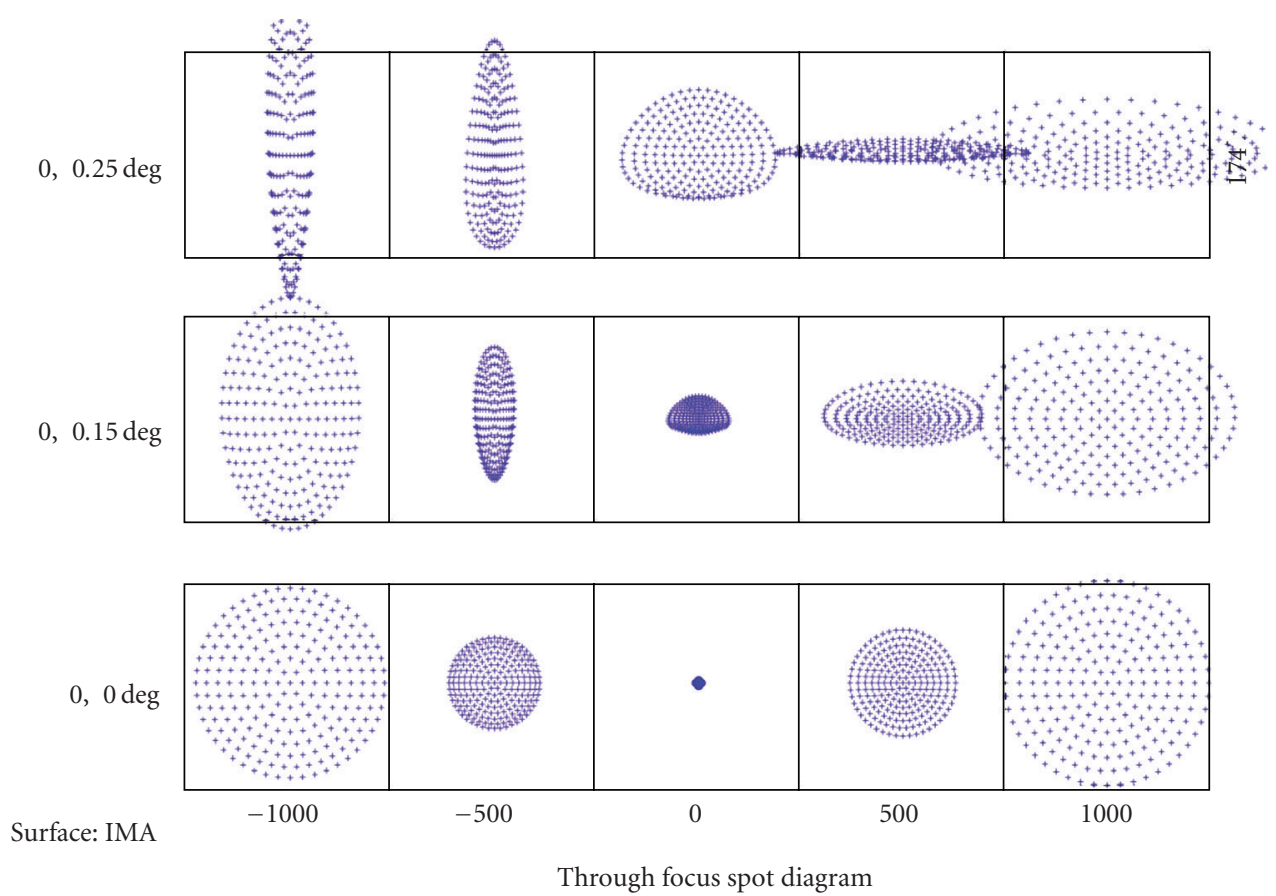

(b)

Figure 4: The optical scheme of the telescope and the relative spot diagram.

5.1. Mechanics. During the conceptual study, different configurations in terms of main structure and subsystem locations have been analyzed to optimize weight and balancing.

The basic solution (see Figure 6) could be a unique hexagonal bench, where all the active (optical payload) and passive (electronics, calibration units, cryogenics) subsystems are mounted. The main structure rely on a monolithic optical bench, reinforced through radial and hexagonal ribs; the structure is closed up by the derotator connection flange which gives the proper continuity to the structure closing the cell, thus enhancing the overall stiffness. Each subsystem will be connected directly to the main bench, then the design and manufacturing of this structure should pay attention to the overall geometrical tolerances. The overall weight obtained from a 1st order dimensioning of this type of structure, in aluminum, is about $715 \mathrm{Kg}$. This instrument set up has the evident advantage of the high accessibility to all subsystems and in addition the space behind M3 is let free and can be used for eventual future add-ons.

Another possible solutions are oriented to minimizing the unbalancing of the overall structure, considering a slightly more compact configuration or a different location of the NIR camera (Figure 7). In this latter case, the NIR subsystem is mounted on a proper truss fixed to the bench; in order to balance the system it is mounted aligned to the main axis of the telescope. The optical beam is directed into the cryogenic system through two pick off mirrors. The other subsystems are mounted on a bench similar to the previous one (hexagonal optical bench with ribs and the closing derotator connection flange). 


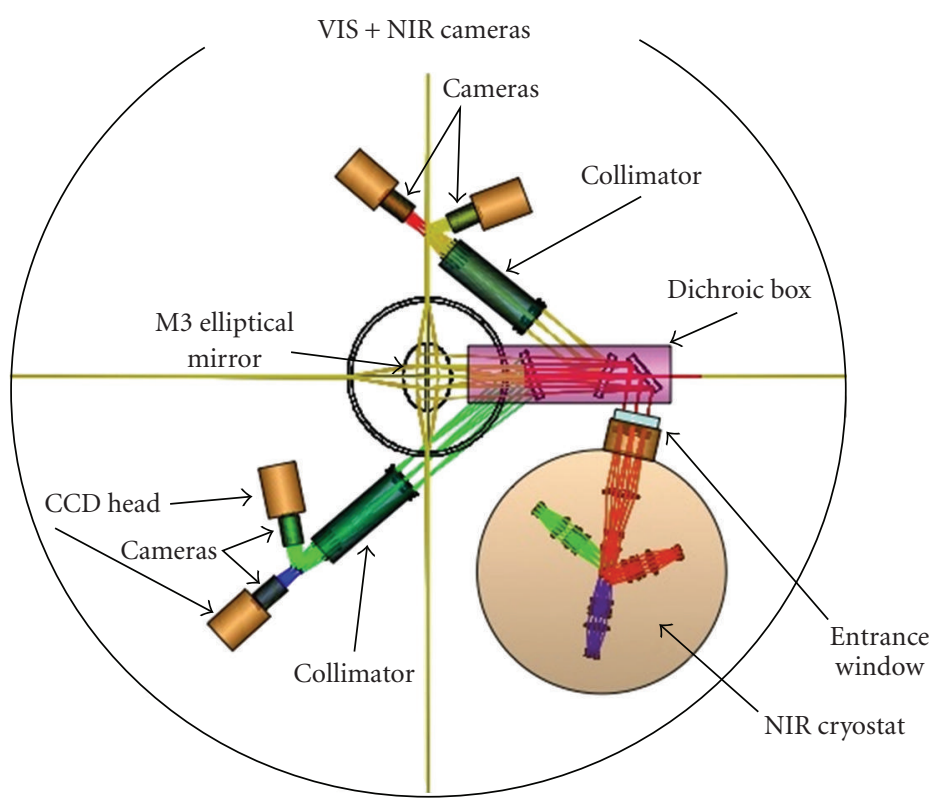

FIGURE 5: The optical scheme of the Pathos focal plane, with the VIS and NIR cameras.

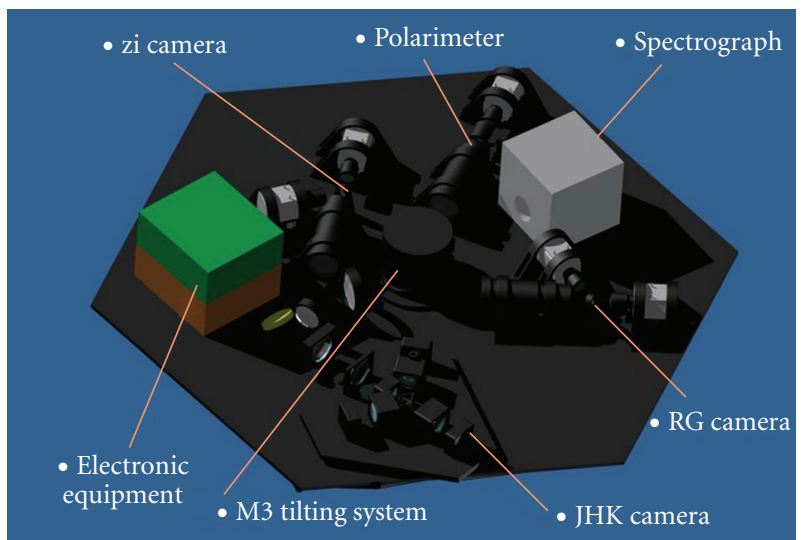

FIgURE 6: The mounting scheme of the Instrumentation at the Cassegrain Focal Plane of Pathos.

This configuration allows a significant reduction of the thickness of both the benches and the ribs. The unbalancing is significant less and can be further optimized by the properly positioning of the electronic systems. The overall weight obtained from a 1st order dimensioning of this type of structure, in aluminum, is about $642 \mathrm{Kg}$. Nevertheless, in this case the accessibility is reduced in particular for the M3 servo system and for all the components, which are under the truss.

5.2. ReD Composite Material. During this case, it has been investigated also the possible gain deriving from the use of advanced materials like Carbon Fiber Reinforced Panels. The results are interesting and will possible drive the following design following also this opportunity. As an example the second configuration made with typical Carbon Fiber Reinforced Plastic will weight around $550 \mathrm{Kg}(-14 \%)$ while if one takes into account an Ultra High Modulus Carbon Fiber Reinforced Plastics it is possible to reach $500 \mathrm{Kg}(-20 \%)$.

\subsection{Optical Design}

5.3.1. The Multi Color Camera (MCC). To allow a simultaneous observation of the scientific target, the MCC configuration will envisage four visible arms, covering the Sloan $g, r, i$ and $z$ bands, and three near infrared arms for the $J, H$ and $K$ bands, simultaneously imaged through a dichroics cascade, as shown in Figure 8.

The lens coating, the dichroics and the filters have to ensure the best overall efficiency, then particular care should be taken in the response curve definition and in the prototyping of the most challenging part, as the two main and larger dichroics.

The MCC will be directly linked to an optical bench at the rear of the telescope, to ensure stiffness and alignment during 


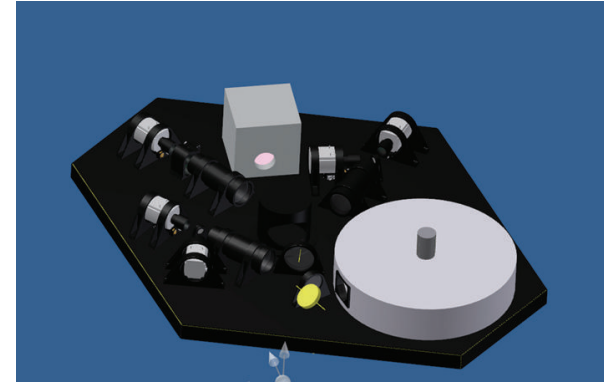

(a)

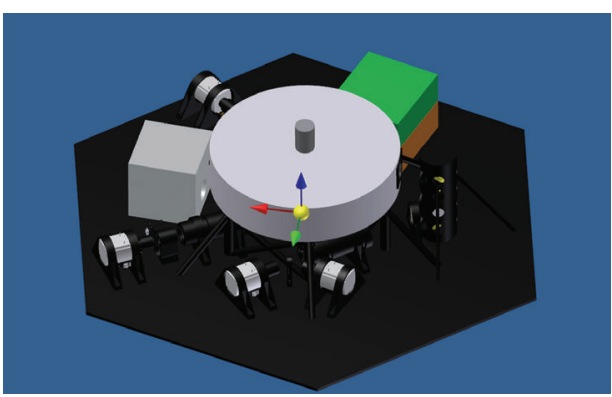

(b)

FIgURE 7: The other different Focal Plane Instrument Configurations.

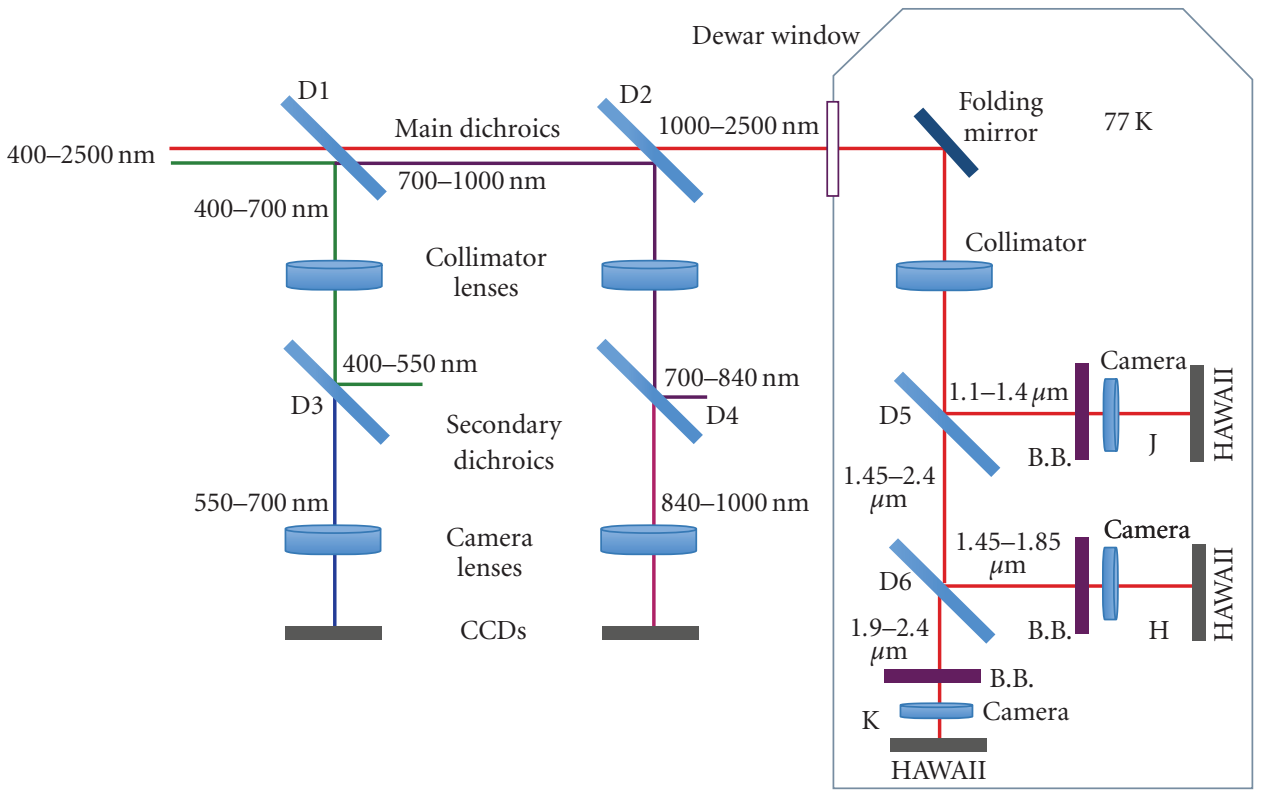

FIGURE 8: The dichroics cascade, feeding the VIS and NIr cameras.

the telescope movements. The configuration of the different parts of the instrument should be optimized also to ensure the best access to the instrumentation for maintenance.

5.3.2. MCC-VIS. The VIS cameras will be devoted to the simultaneous observation of the same field of view at four different bands. At least three different dichroics are needed to split wavelengths. They can be put both at a convergent (or divergent) beam, or in a collimated beam. Requirements onto field of view and pixel scale ask for focal reducer system. The large field of view cannot be achieved through classical corrector lenses just in front of the telescope focal plane, so a collimator-camera system is the only choice. Then, some of the dichroics can be put within the focal reduce elements in order to reduce its size and complexity, gaining in space envelope for the overall instrument suite at the same time. So we chose to put a dichroic (D1) in the convergent beam coming from the telescope secondary mirror, and making the second wavelength split in collimated beam (D3 and D4). The other large dichroic D2 is used to split VIS and
NIR light, feeding the NIR cameras. The minimum pixel scale to give good seeing sampling should be large than $3 \mathrm{pixel} / \mathrm{arcsec}$. To cover a $10 \times 10 \mathrm{arcmin}$ onto a $2 \mathrm{~K} \times 2 \mathrm{~K}$ CCD detector area, the best compromise can be reached with a $3.4 \mathrm{pixel} / \mathrm{arcsec}$ plate scale. Because the telescope focal ratio is about $F / 10$, an $F / 10-F / 3.5$ focal reducer is needed. Lens solution classes will give the best performances in term of efficiency, cost and image quality, within a limited space envelope, in order to cope with many arms within the same optical bench assembly. A possible optical design has been done, meeting all those requirements. Only spherical lenses, with maximum diameter of $150 \mathrm{~mm}$, have been adopted, in order to keep overall cost and manufacturing time low. The layout below shows rays crossing all the elements, starting from the telescope focal plane up to the CCD detector plane. Dichroics and filters will be placed near the image pupil of the telescope (in the middle). All the optical train is $70 \mathrm{~cm}$ long, and the collimated beam have a $40 \mathrm{~mm}$ diameter (Figure 9). This same design will perform very well at all visible (400-1000 nm) wavelengths. This means that 


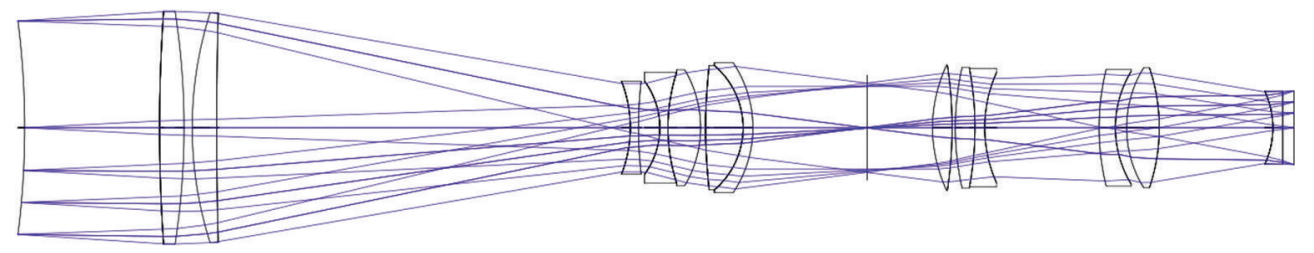

FIGURE 9: The basic optical scheme for the VIS cameras.

multiple arms can be built with just two identical collimators and four identical cameras, decreasing cost and alignment quite a lot.

5.3.3. MCC-NIR. Following the same ideas developed for the VIS channels, NIR wavelengths are split via two dichroics, placed into a collimated beam. A NIR optimized collimator camera system will match the telescope focal ratio to the NIR detector pixel size. Special infrared glasses will been used, to optimize both transmission and image quality. First collimator lens can be used also as cryostat window, reducing the number of air-glass interfaces. In this case, first collimator window will be warm, while all following components will be cooled to $77 \mathrm{~K}$ to reduce thermal background, especially in the $K$ band. Also dichroics will be optimized to work at low temperatures. To better define spectral bands (JHK), also multilayer filters will be used. A camera prototype will be set up to understand if we need a cryogenic focusing system onto each channel, to improve image quality. This can be possibly realized with a moving lens into the camera assembly, or at the detector level. A proper trade off will be done to make a decision. The $K$-band channel will suffer from thermal emission, then a dithering system will be added, to reduce this effect. A possible solution is to add a folding mirror into the camera assembly that can be moved with cryo motors.

\subsection{Ancillary Instruments}

5.4.1. Spectrograph. The spectrograph has been designed with the following parameters:

(i) $R=800$,

(ii) CCD: $1 \mathrm{~K} \times 1 \mathrm{~K}, 13$ um pixel, BI DD (e.g., Princeton Pixis),

(iii) Slit: 1 arcsec $\times 4$ arcmin,

(iv) Sampling: 4.4 pixel (spectrally), 4.0 pixel/" (spatially),

(v) Coverave: 3700-9200 Angstrom (in three exposures),

(vi) Efficiency: 45\% (peak, from entrance slit to detector, including DQE),

(vii) Movable functions: grating, maybe focus (camera/collimator).

In Figure 10 the optical scheme of the spectrograph and the relative spot diagram are shown. As you can see, the dimension of the spectrograph are relatively small (about $30 \mathrm{~cm}$ ) and the only movable part is the grating, mounted onto a rotating stage.
5.4.2. PhotoPolarimeter. The polarimeter designed for the CODEVISIR telescope has the following main parameters:

(i) a field around $4^{\prime} \times 4^{\prime}$,

(ii) a wavelength coverage in the visible band (between 400 and $800 \mathrm{~nm}$ ),

(iii) four channels relative to the four Stokes parameters.

Basic scheme of the polarimeter is the classical "collimator/camera" system.

The incoming beam is collimated through lenses. The collimated beam is split into four beams through a combination of Wollaston prisms. This combination takes the name of WeDoWo (WEdged DOuble WOllaston) [4]. Following the behavior of the imaging instrument, the aim of WeDoWo is the simultaneous production of four different beams, each one containing the information of one of the Stokes polarization vector parameters (Figure 11).

Each beam is then focused through a camera into the detector. The main possibility is to drive all the four beams into the same detector.

Deeper study arises from the filtering option, where the possibility to cover all the visible spectrum and part of the very near infrared becomes interesting. A filter wheel will be placed in the parallel beam, with at least three positions (standard UBV filters) and an empty slot for white-light observation mode.

One of the auxiliary tasks of the polarimeter can be the guiding system. Indeed, recombining the four channels there will be the possibility to have an immediate field monitor. In principle, due to the small probability of an high polarized guide star, there is the possibility to acquire in real time only one of the four channels, depending on the speed requirements.

5.5. Cryogenics. Differently from visible CCD detectors where only the internally generated dark current must be limited by active cooling, IR detectors working beyond 1$2 \mu \mathrm{m}$ are sensitive also to the possible thermal background generated by surrounding surfaces. A limitation to this effect requires the insertion of actively cooled shielding surfaces.

In the past years, large experience has been achieved in the cryogenics field and, in particular in the astronomy field, this is a common practice for this kind of instruments. Then, standard solutions exist, that allow to cool down and keep them cold for a long period, with high thermal stability and reliability. These include cryocoolers constituted by a main compressor linked through a high pressure $\mathrm{He}$ line to a smaller cold head, that eventually provide the cyrogenic 


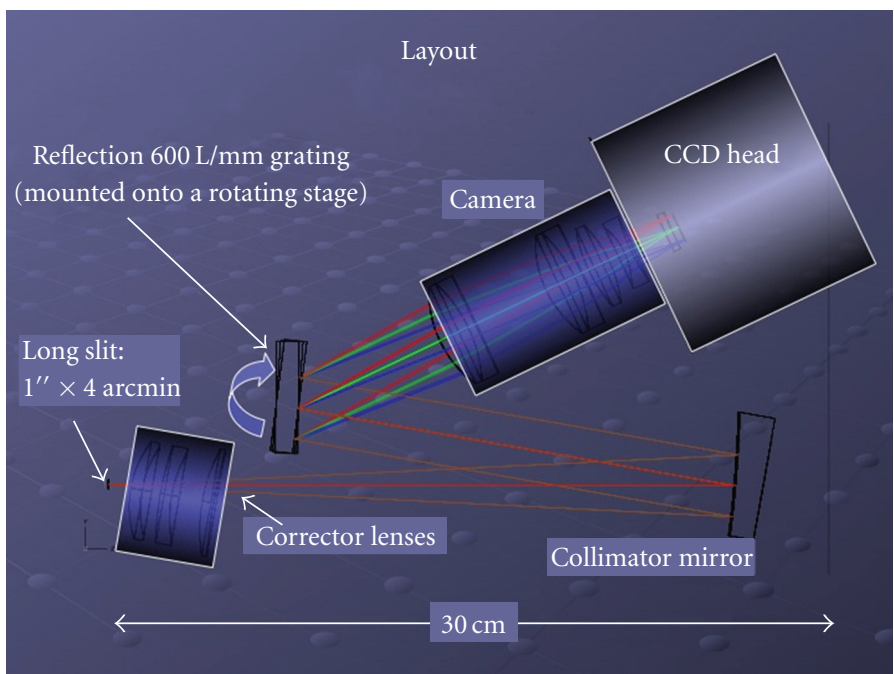

(a)

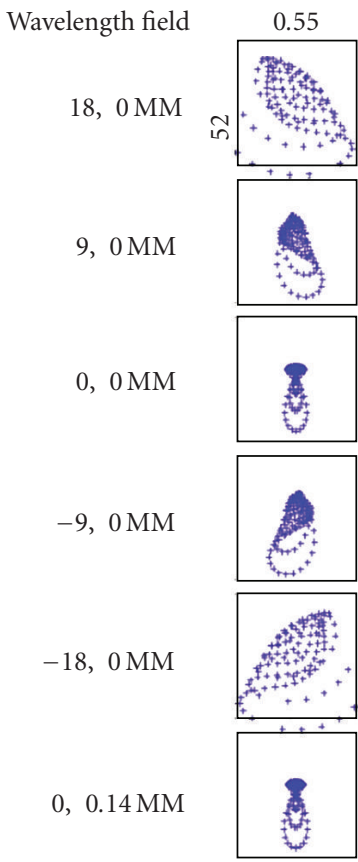

Surface: IMA

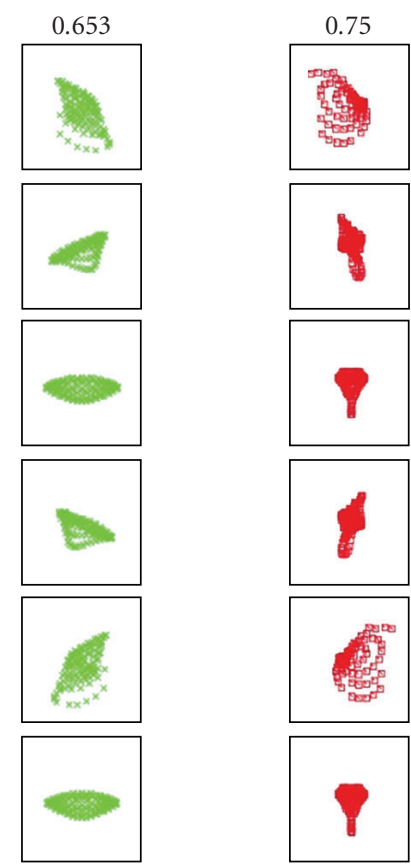

Matrix spot diagram

(b)

FIGURE 10: The optical layout of the spectrograph and the relative spot diagram at 550, 653 and $750 \mathrm{~nm}$.

temperature to the instrument. These cryocoolers can easily have cryogenic power of tens of Watts@77 K (for near infrared instruments). These have been extensively used at the focal plane of telescopes and definitively provided a stable and reliable system.

The current design is based on the Leybold CoolPower 5/100T Cryocooler, a Dual Stage Cold Head that can provide up to $100 \mathrm{~W} @ 80 \mathrm{~K}$ at the 1st stage and $35 \mathrm{~W} @ 40 \mathrm{~K}$ at the 2 nd stage. The basic scheme of the cryostat (see Figure 12) envisage an optical bench, on which all the optomechanical elements and the NIR arrays rely, surrounded by two radiation shields, to avoid the direct irradiation from the external $300 \mathrm{~K}$ of the external temperature. As usual, a set of standard devices will be hosted in the cryostat, as a Lyot stop (to shield the thermal diffuse light), temperature sensors (to monitor the temperature of the most critical points of the cryostat, as the arrays and the optics), charcoal getters (with their own heaters), heaters (to fine tuning the bench temperature), a dithering mirror and a moving shutter (to perform dark current measurements).

Besides the standard solutions, we will investigate the possible use of mini cryocoolers, that essentially use the same 


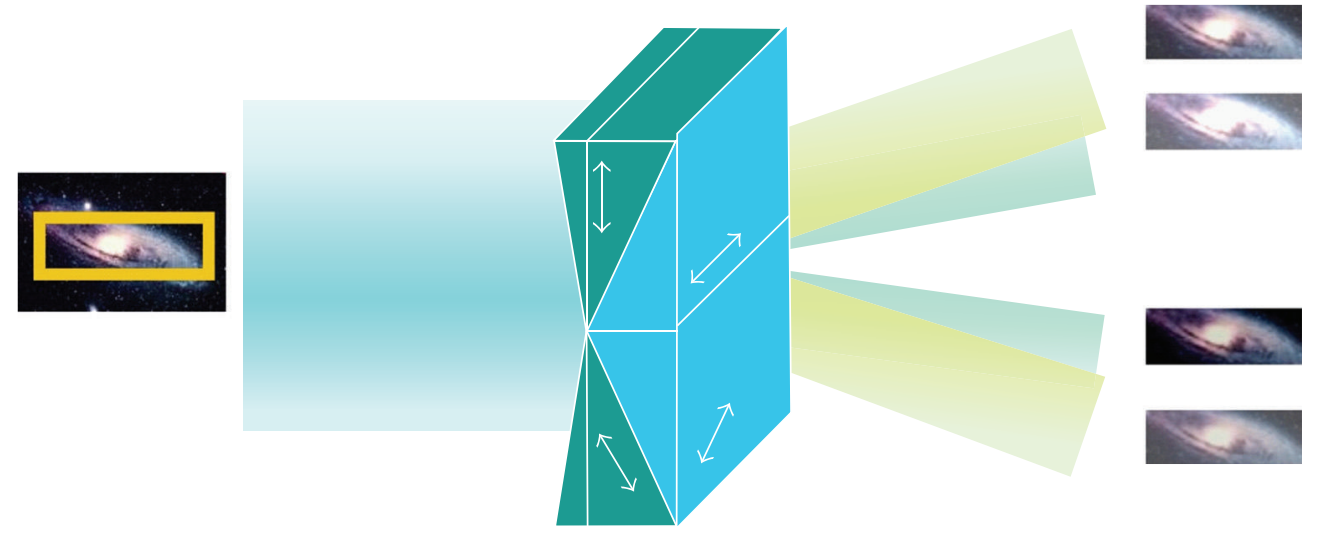

FIgure 11: The Wedowo scheme for the Pathos Spectrograph.

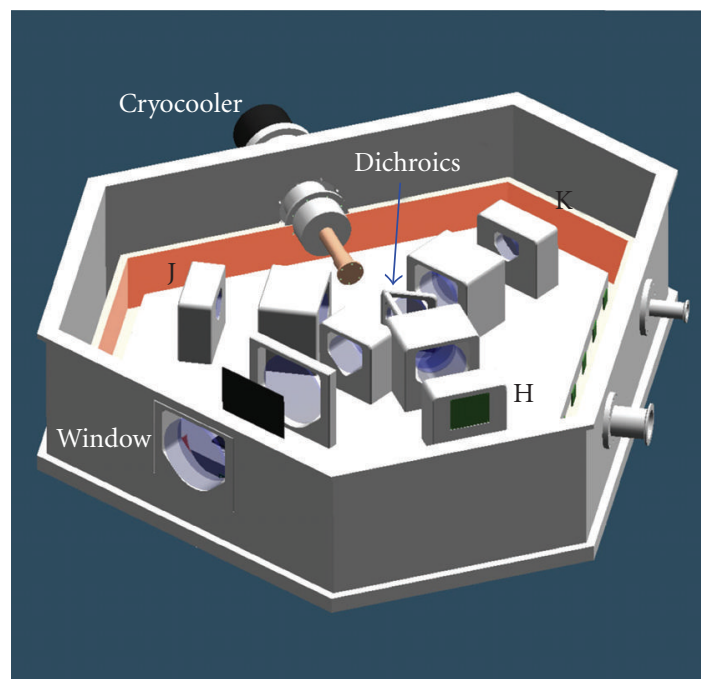

FIGURE 12: The cryomechanical scheme for the NIR camera.

cryogenics concepts but have very small and compact sizes: this would allow to have all the cryogenics device directly at the focal plane of the telescope, thus avoiding the long He line needed for the standard cryocoolers. A fast moving telescope would certainly have benefit of that. Of course, the reduced dimensions reflect in reduced cryogenic power, then pushing the instrument design toward the most compact and light instrument. Anyway, the recent experience in this field with REMIR@REM showed that these devices are sensitive to the telescope movement and then a deep investigation of their performances and a tight collaboration with industry are needed to assess their use at the telescope.

In order to improve reliability, the possibility to avoid the use of servo system into the cryogenic environment will be investigated: this means that the optics should be manually aligned before the cooling and adjust after following heating up cooling down cycles. If this can be achieved there is no needing of differential focus adjustment between the NIR arms because the cryogenic environment is a thermoelastic stable condition. In addition it will be investigated the opportunity of stabilize the temperature inside the whole instrument set-up. Doing so the thermo-elastic strain can be eliminated and the focus of each arm can be done directly by a piston motion of the secondary mirror of the telescope. The investigation of this issue will open the possibility of the reduction of the servo-systems, but must be done carefully making a costs versus benefits analysis.

\subsection{Detectors}

5.6.1. Visible. The VIS image detection system is based on a set of four CCD cameras with $2 \mathrm{~K} \times 2 \mathrm{~K}$ class detectors. Due to the relatively high background present in the VIS observations (broad-band imaging) the use of autonomous thermoelectrically cooled cameras seems to be the most appropriate solution. With such a choice we will avoid the insertion of more complicated cooling systems (liquid nitrogen or cryo-coolers) in favor of a simple water recycling system.

There is presently an extremely wide market for commercial cameras mounting customized scientific detectors and operating in cooled mode inside a windowed thermally insulated chamber. Even overall dimensions and resulting weight is reduced when compared to classical cryostat based cameras serviced by modular control electronics. Maximum of compactness and system efficiency, even in terms of spare parts, will be obtained making use exactly of the same camera model to populate VIS allowing diversification only for the detector QE specifics in the four bands.

The present reference choice is among two quite similar thermoelectrically cooled cameras (Princeton Pixis2048, in Figure 13, and Andor iKon-L 936) both allowing the insertion of $2 \mathrm{~K} \times 2 \mathrm{~K} \mathrm{CCD}$ detectors (all the standard E2V scientific set with different coating and substrate options is available) with cooling capabilities below $-60^{\circ} \mathrm{C}$. Insertion on the informatics network taking care of detectors is simplified by the availability of fiber-based USB interfaces.

5.6.2. Near Infrared. At the time of writing, the best NIR detectors available in the market for astronomy are from Teledyne and Raytheon companies, being the former better 


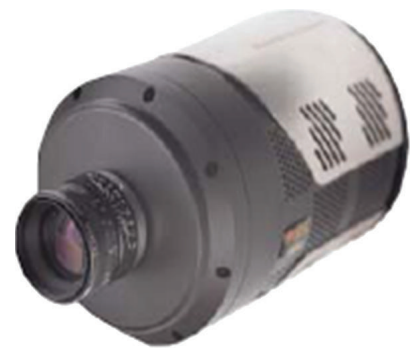

(a)

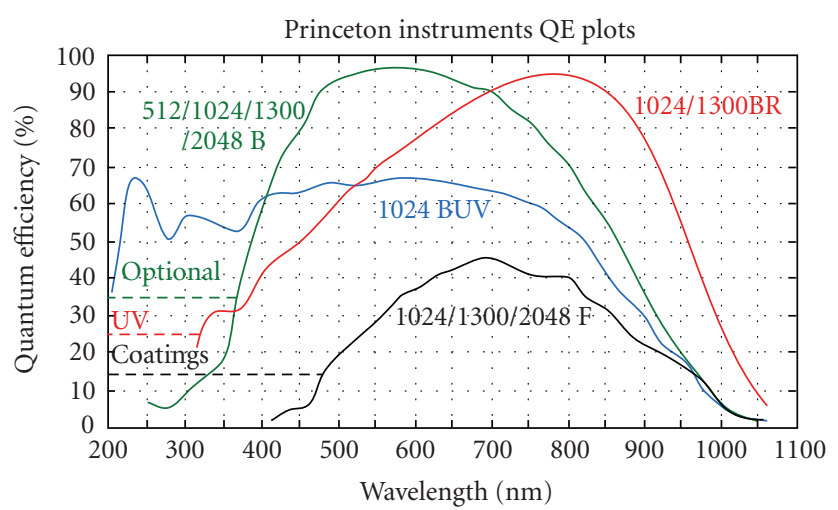

(b)

FIgure 13: Princeton Pixis thermoelectrically cooled cameras and QE curves for the standard set of E3V scientific detectors available.

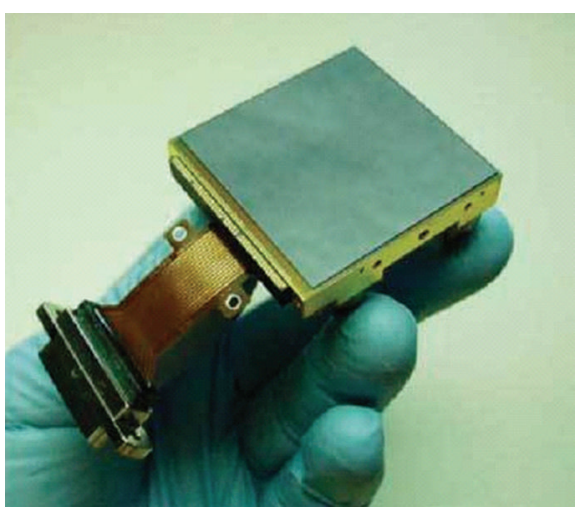

(a)

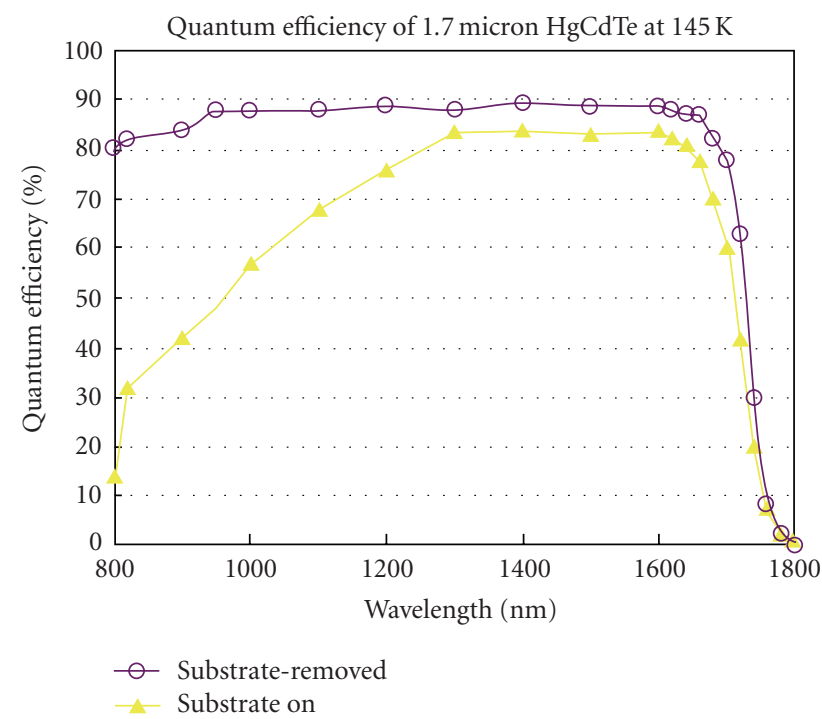

(b)

FIGURE 14: The Hawaii-2RG IR array from Teledyne and the predicted QE for $1.7 \mathrm{~mm}$ cut-off wavelength. With the substrate removal, the QE curve extends toward the visible domain [5].

in terms of noise and can be delivered together with its own cryogenic control electronics. Anyway, the rapidly evolving technology in the field of NIR detector suggests to be careful in the choice of the final detector and to follow all the evolution of the market in this field. Currently, we are oriented towards the Teledyne Hawaii-RG series of detectors (Figure 14), that can be delivered in different format $(1 \mathrm{k}$ $\times 1 \mathrm{k}, 2 \mathrm{k} \times 2 \mathrm{k}$ and even $4 \mathrm{k} \times 4 \mathrm{k}$ pixels) and different wavelength coverage (cut-off at 2.5, 1.7 and $5.3 \mu \mathrm{m}$ are standard), allowing a certain flexibility in the choice of the best detector for each photometric band. These detectors shows the better performances in terms of dark current $\left(<0.01 \mathrm{e}^{-} / \mathrm{s} /\right.$ pixel @ $90 \mathrm{~K}$, for a $2.5 \mu \mathrm{m}$ cut-off wavelength), Read Out Noise (13-15 $\mathrm{e}^{-}$with Single CDS, down to $<3 \mathrm{e}^{-}$ with multiple sampling) and Quantum Efficiency $(>80 \%)$ $[5,6]$.
5.7. Control Electronics. Teledyne is offering a reliable and performing cryogenic electronic for its NIR hybrid detectors, the SIDECAR ASIC, designed as a compact focal plane electronics on a single chip for Teledyne focal plane arrays HAWAIIs, including the $4 \mathrm{k} \times 4 \mathrm{k}$ and the forecoming $8 \mathrm{k}$ $\times 8 \mathrm{k}$ [5]: it supports all possible modes of operation and can be used in the same cryogenic environment of the detector, thus minimizing the control electronics that generally accompanies such kind of devices (see Figure 15). ESO staff succesfully tested this electronics and they are planning to build their own interface for the SIDECAR ASIC (replacing the JADE2 card) and to include the SIDECAR in the standard VLT and E-ELT software environment [7].

Moreover, in principle it could be used also to drive CCDs: very few groups around the world are involved to this aim but it would be worth to open a R\&D in this field 


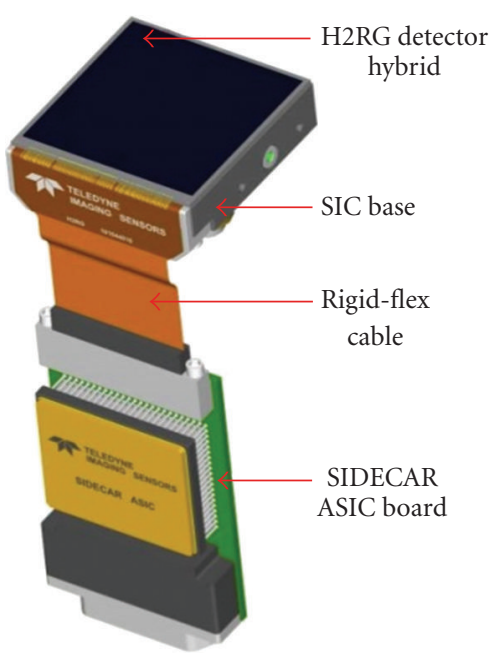

(a)

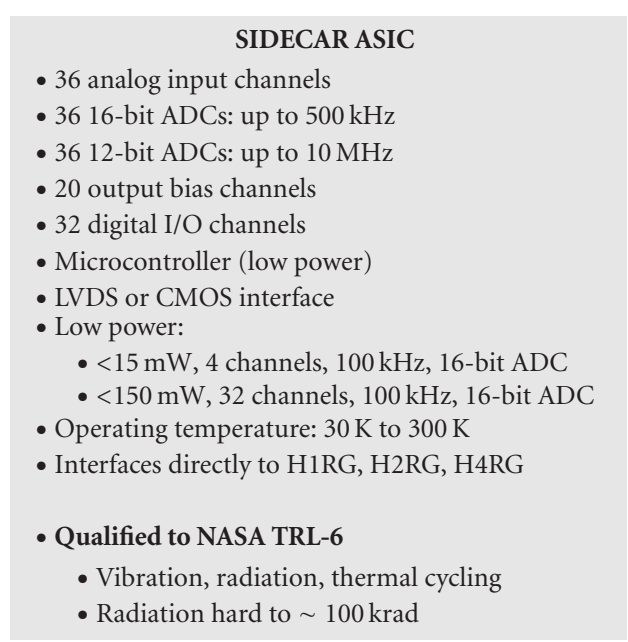

(b)

Figure 15: The new H2RG-SIDECAR cryogenic packaging and the main characteristics of the SIDECAR electronics.

because the possibility to have a single control electronics for all the detectors in Pathos would increase the reliability of the overall system, even if at higher costs with respect to the standard CCD reading electronics.

\section{Control Software and Data Management System}

The development of the control software and Data Management System (DMS) will follow an approach similar to that adopted for REM ([1, 8] and reference therein). Operations of the whole system will be managed by a set of processes each one dedicated to a very specific task and in constant inter-communication. A dedicated process will then act as a coordinator of all the above activities.

However some components will require major changes and improvements in order to satisfy all the requirements of the project in terms of (1) safety, (2) reliability, (3) effectiveness. In particular the huge amount of data (scientific and calibration frames, telescope and instruments telemetry, meteo, etc.) will require the DMS to rely on

(1) reliable internal and external communication network,

(2) reliable redundant sub-systems,

(3) relational databases,

(4) multithread processes using a client/server architecture.

Figure 16 shows the overall s/w architecture. The two core components are the "Observing System" at the telescope site and the "Supervisor System" at a remote site in Italy. The various components will communicate mainly through TCP/IP sockets with processes implementing a client/server scheme. The Observing System will save all the data in a local DB system and will perform all the low-level data reduction.
Data will then be transferred to Italy (in a synchronous or delayed manner) where all the high-level processing will be performed. The general users will have access to the data stored in the latter DB server through an internet accessible server using several types of interfaces ensuring full flexibility. The DB server will be mirrored at least at two sites.

This project has been designed with the explicit main goal to manage unexpected alerts from many sources. There is a large family of astrophysical objects potentially providing events occurring on rapid timescales, that is, comparable or shorter than the typical telescope pointing time (about 30$60 \mathrm{~s}$ ) as GRBs, XRBs, high-energy transients of various sort, and so forth. We do not discuss here the astrophysical interest of rapid observations of these objects, however it is the case to remind us that optical and near-infrared observations carried out on the occurrence (or with a delay of a few tens of seconds) of high-energy events with a telescope of adequate collecting area are a real novelty and will effectively open a new observational window.

Alerts from astrophysical transient phenomena will likely still come when the telescope will be operational via the GCN system. However, other specialized alert delivering systems already existing (i.e., IBAS, etc.) or future available must also be considered (from neutrino telescopes, extensive air shower experiments, gravitational wave detectors, etc.). In addition, alerts will also be received for transients singled out by other optical instruments, as high-time resolution wide-field telescopes monitors or other robotic telescopes. The management of these families of alerts will be driven by a devoted software component. In general the delivering of these alerts will follow private standard solutions, developed in-house by the missions or experiments delivering them. This code must therefore implement all these solutions and be easy to update and enriched by new alert sources and/or technical solutions which can with time become available. All the received alerts will feed a database and a Figure of Merit (FoM) will be derived. The FoM, essentially, is a priority 


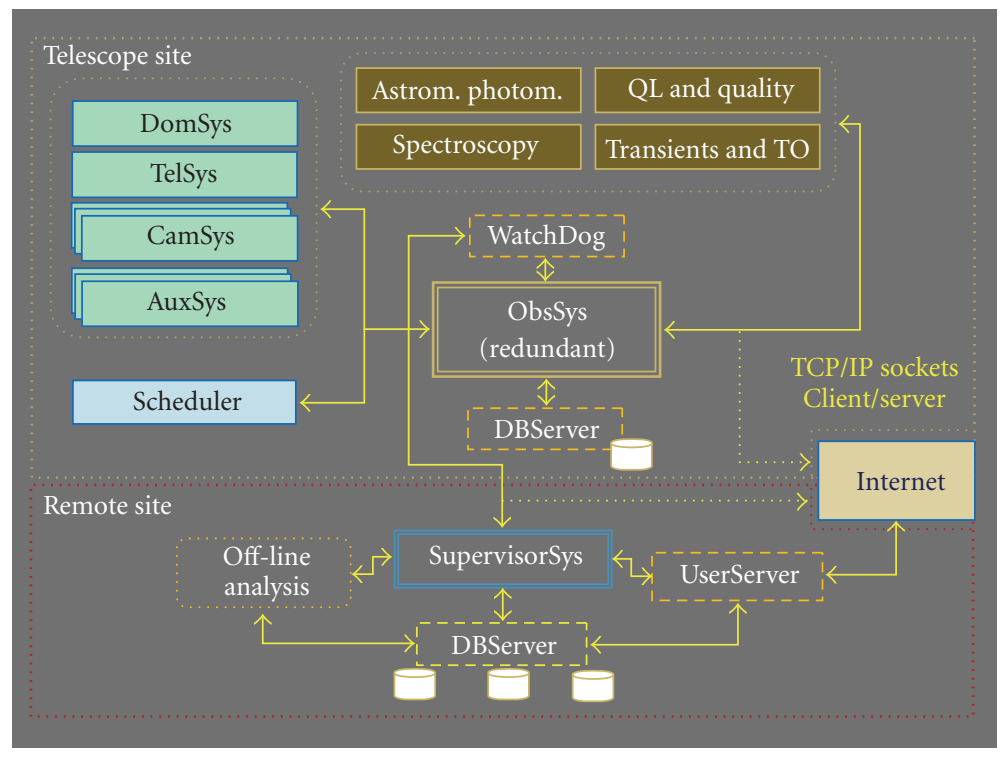

Figure 16: A schematic view of the control s/w and DMS.

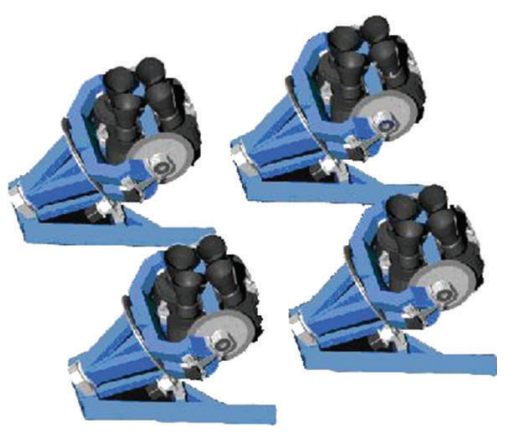

SITE A

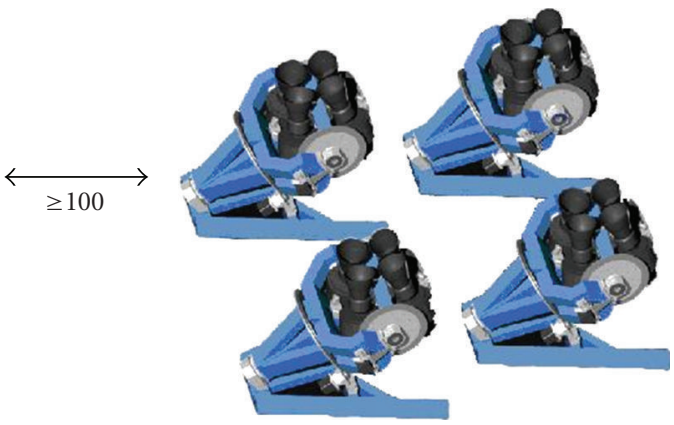

SITE B

FIGURE 17: Schematic view of architecture of the Wide-Field array.

evaluation based on a multiparametric approach as in the case of the Swift satellite. The final output will be a grade to be compared to the grade of the observation possibly already in progress by the telescope. The FoM will also allow the telescope management system to evaluate a priority among possible multiple subsequent alerts.

\section{Wide Field Array}

Pathos will be equipped with the most advanced visible Wide Fieled Array ever built, capable to trigger the Pathos observations of rapidly variable phenomena ( $\mathrm{SNe}$ and GRBs), in absence of satellite $\gamma$ and X-ray triggers for GRBs. The self-triggering capability will be secured by a construction in collaboration with the "Pi of the Sky" group [9] of a dedicated wide-field (WF) array. Here the main challenges are to considerably shorten the time scale with which the sky will be probed, as compared with already existing projects, and to develop a concept of autonomous CCD camera equipped with sufficient computing power, memory and GBethernet link as to serve as independent unit capable of performing at least primary image processing and analysis, discover candidates for optical transients and exchange corresponding information with other such cameras and wide-field arrays present in the network.

The envisaged architecture assumes 2 sites located at a base distance of about $100 \mathrm{~km}$, as shown in Figure 17. Each site should be equipped with 16 CCD cameras, covering about 2 sr of the sky. Depending on settings, cameras from both sites will work pair wise in coincidence or observe separate FOV's. Note that for short optical transients, elimination of background due to cosmic radiation requires observation of the same FOV by at least 2 units. In addition, sufficiently long baseline allows to eliminate Sun reflections from military, maneuvering satellites with orbital elements which are kept secret to civilian community. In order to filter out background and ultimately to select transients of astrophysical origin in the real time requires development and implementation of very efficient and clever algorithms. 


\section{Dome}

The requirement on the pointing rapidity of the telescope, force us to find a proper solution for the dome, that should follow the velocity of the telescope. Nevertheless, other issues linked to the dome choice have to be taken into evaluation, as feasibility, costs, wind and dust shielding, proper thermalization.

Different dome configurations will be taken into consideration (horizontal or vertical slit, boxes, geodesic, fully retractable) and some simulations have been done to evaluate the impact of the incoming wind on the dome.

\section{Conclusions}

The evidence that the GRBs are, on average, weaker than thought before, suggested us to exploit our precious experience made with the robotic $60 \mathrm{~cm}$ REM telescope in the last 6 years and investigate the possibility to build and operate a larger telescope. We are now completing a feasibility study to build and operate a robotic $4 \mathrm{~m}$ class telescope, equipped with a multichannel VIS-NIR camera, able to point on the triggered target in less than 50-30 s.

\section{Ackowledgments}

The authors wish to thank MIUR (Italian Ministry of Research) which contributed to the construction of REM and to the present feasibility study, ASI (Italian Space Agency) for its contribution to Swift. The authors would like to thank the ESO La Silla staff, who in the past years collaborated with them in setting up and maintaining the REM telescope and its instrumentation. They would like to thank also Maciej Sobczak, for his useful and skilled software advising.

\section{References}

[1] E. Molinari, S. Covino, F. D’Alessio, et al., "REM, automatic for the people," to appear in Advances in Astronomy.

[2] E. Molinari, S. D. Vergani, D. Malesani, et al., "REM observations of GRB060418 and GRB060607A: the onset of the afterglow and the initial fireball Lorentz factor determination," Astronomy \& Astrophysics, vol. 469, pp. L13-U16, 2007.

[3] J. L. Racusin, S. V. Karpov, M. Sokolowski, et al., "GRB 080319B: a naked-eye stellar blast from the distant universe," Nature, vol. 455, no. 7210, pp. 183-188, 2008.

[4] E. Oliva, "Wedged double Wollaston, a device for single shot polarimetric measurements," Astronomy \& Astrophysics, vol. 123, pp. 589-592, 1997.

[5] J. W. Beletic, R. Blank, D. Gulbransen, et al., “Teledyne imaging sensors: infrared imaging technologies for Astronomy \& Civil Space," in High Energy, Optical, and Infrared Detectors for Astronomy III, vol. 7021 of Proceedings of SPIE, Marseille, France, June 2008.

[6] G. Finger, R. Dorn, L. Mehrgan, M. Meyer, and J. Stegmeier, "Evolution of IR detection and possible outcomes for astrophysics," EAS Publications Series, vol. 37, pp. 343-353, 2009.

[7] R. J. Dorn, S. Eschbaumer, D. N. B. Hall, et al., "Evaluation of the Teledyne SIDECAR ASIC at cryogenic temperature using a visible hybrid H2RG focal plane array in 32 channel readout mode," in High Energy, Optical, and Infrared Detectors for Astronomy III, vol. 7021 of Proceedings of SPIE, pp. 70210Q1-70210Q, Marseille, France, June 2008.

[8] M. Stefanon, S. Covino, D. Fugazza, et al., "The REM observing software," to appear in Advances in Astronomy.

[9] K. Malek, T. Batsch, M. Cwiok, et al., "“Pi of the Sky” detector," to appear in Advances in Astronomy. 

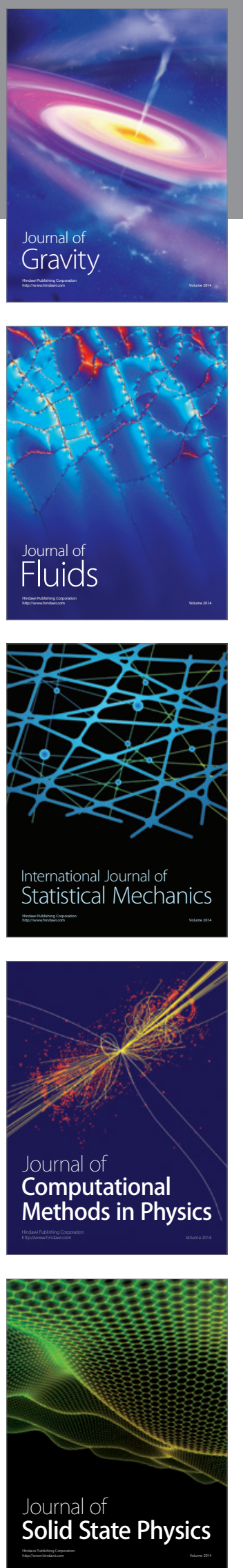

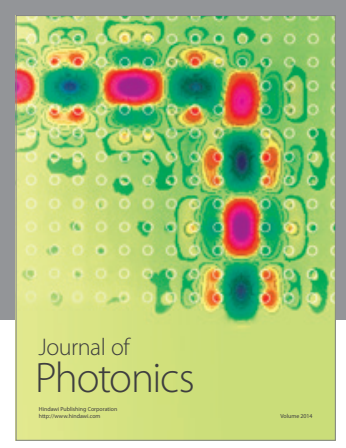

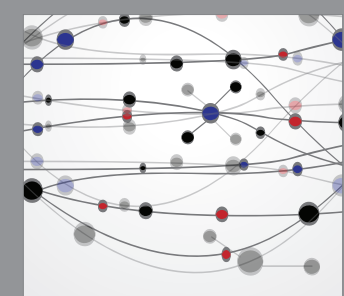

The Scientific World Journal
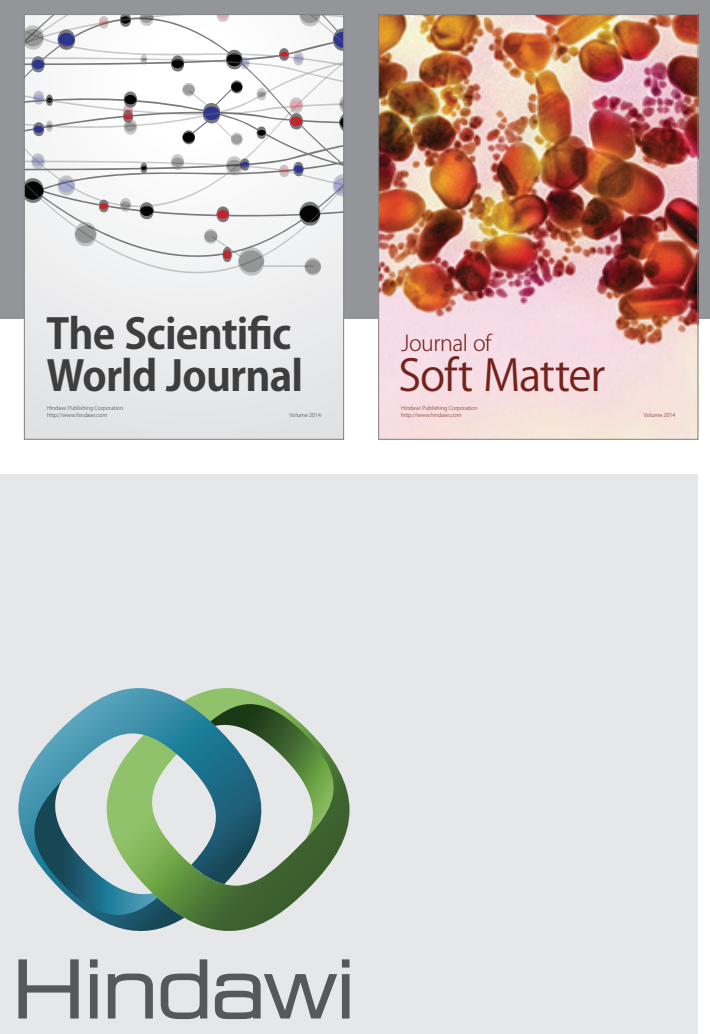

Submit your manuscripts at

http://www.hindawi.com
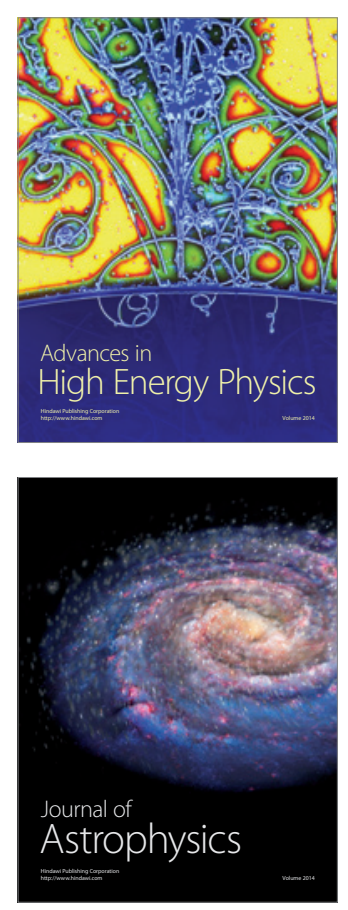
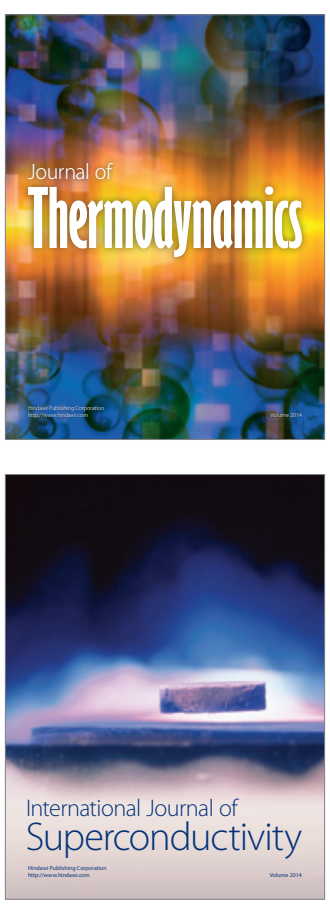
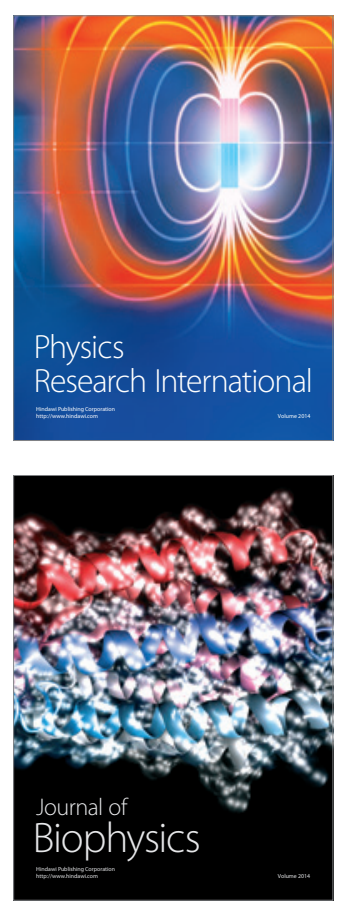
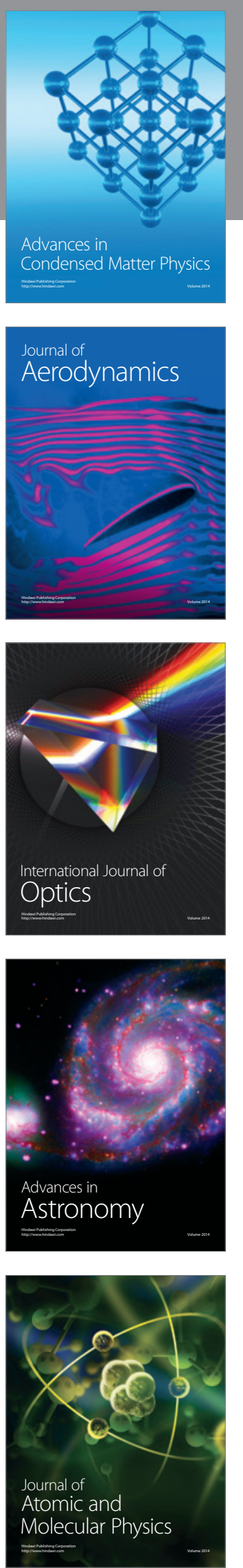